\title{
Cladding modes FBG curvature sensor based on a core misaligned splice
}

\author{
C. Jesus ${ }^{\mathrm{a},}{ }^{,}$, P. A. S. Jorge ${ }^{\text {a }}$, J. M. Baptista ${ }^{*, a}$, O. Frazão ${ }^{\text {a }}$ \\ ${ }^{a}$ INESC-Porto, Rua do Campo Alegre, 687, 4169-007 Porto, Portugal; \\ *Centro de Competências de Ciências Exactas e de Engenharia, Universidade da Madeira, Campus \\ da Penteada, 9000-390 Funchal, Portugal.
}

\begin{abstract}
A novel fiber optic curvature sensor based on a core-offset single-mode fiber (SMF) and on a fiber Bragg grating (FBG) is presented. The FBG cladding modes are efficiently excited by the large core misalignment. The curvature of the beam can be obtained by the reflected power of the core mode and the recoupled cladding mode.
\end{abstract}

Keywords: Bragg grating, cladding modes, bending measurement.

\section{INTRODUCTION}

Fiber Bragg grating (FBG) sensors have generated great interest in recent years because of their many industrial and structural health monitoring applications. Based on diffraction mechanism, the fiber grating can be used as fiber mirror or filter for fiber optic sensing or communications. FBG sensors have been widely used for strain and temperature measurement [1].

Several configurations have been proposed for measurement of curvature using FBGs. An etched chirp FBG was used by $\mathrm{Yu}$ et al [1]. The bent radius was measured by the reflected power of the grating. This technique is simple but needs a power reference, and is also known that the etching induces fragility in the structure. Other works were reported using a tilt FBG (TFBG). The transmitted spectrum of a TFBG is composed of several discrete modes which corresponds to two different mechanisms, the modes at the longest wavelength come from the self coupling of the core mode while the others are due to the backward coupling with the cladding modes. The high effective index cladding modes are sensitive to curvature and their amplitudes decrease as a function of bent radius. Caucheteur et al [3] presented a TFBG for simultaneous measurement of bending and temperature. The curvature was measured though the amplitude of the curvature sensitive cladding modes and the temperature from Bragg resonance. This configuration uses spectral interrogation and typically the cladding modes of TFBG require transmission readout. Coupling light from core mode to the cladding mode in the FBG forward direction enables the reflection readout operation mode. Recently some authors explored this possibility. Using a short MM fiber section, Jin et al [4] showed a reflection operation of a TFBG configuration for curvature sensing. Based on the same principle and on the curvature sensitivity of the TFBG a core offset section was explored by Guo et al [5] and Huang et al [6] for vibration sensing. Recently an LPG/TFBG hybrid sensing head was also studied by Shao et al [7]. In this case, due to LPG sensitivity to bending, both reflected peaks are affected by the measurand, and the difference of the power was used to obtain the curvature. These configurations use TFBGs, which cladding modes are spectrally not so clear and almost all of them use spectral interrogation.

In this paper we present a high reflectivity FBG with a core-offset-splice in a single mode optical fiber. This technique enables the possibility to readout in reflection the cladding mode and the core mode. Is well known that the core mode is not sensitive to bending, while the optical power of the cladding mode is affected. The analysis of both modes allows a self-referenced measurement of curvature. Since the measurement is independent of the wavelength of the peaks a filter and two photodetectors were used for signal detection.

\section{CHARACTERIZATION OF THE SENSING HEAD}

The sensitive system is formed by a high-reflectivity FBG $(\mathrm{R} \approx 98 \%, 6 \mathrm{~mm}$ in length). The grating has been written in a hydrogen loaded SMF-28 fiber by UV beam scanning phase mask technique. The fiber segment containing the grating was cleaved approximately $5 \mathrm{~mm}$ distance from the FBG. Another long piece of fiber previously connected to an optical

21st International Conference on Optical Fiber Sensors, edited by Wojtek J. Bock, Jacques Albert, Xiaoyi Bao, Proc. of SPIE Vol. 7753, 77538U · C 2011 SPIE · CCC code: 0277-786X/11/\$18 · doi: 10.1117/12.886029 
circulator was cleaved. Both cleaved fibers were placed in to a splice machine. To monitor the spectra behavior and optimize the cladding modes the splice was real time monitored in reflection using an Advastest Q8384 Optical Spectrum Analyzer. In order to obtain a dominant cladding mode a lateral core-off-set was induced. In the distal end of the sensing head, an index matching gel was used to avoid undesirable reflections. Figure 1 shows the proposed configuration and two different types of mode recoupling mechanism. The first one (Figure 1.A) the guided light is coupled to the backward cladding mode by the FBG and is recoupled to the guided mode by the core-lateral-offset section. The second one (Figure 1.B) the guided light coupled from core to the cladding modes by the misaligned section is recoupled to the backward guided mode by the FBG [8].
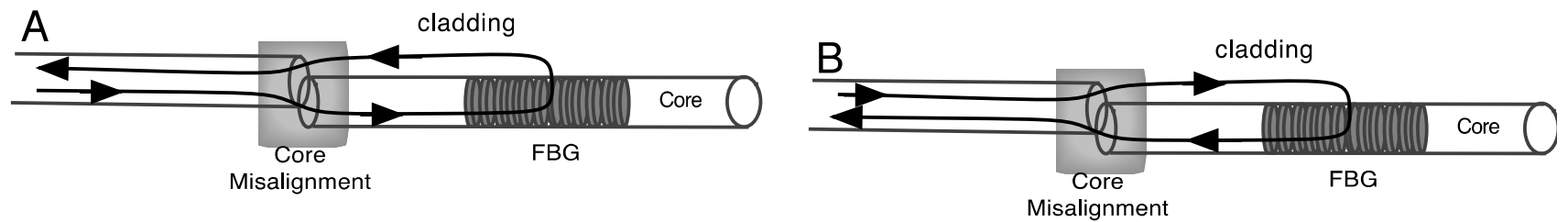

Figure 1 - Sensing head formed by a high reflectivity FBG and a misaligned core splice. The two recoupling mechanisms are described in $A$ and $B$.

Figure 2 shows the sensing head with the misaligned splice, where the lateral-core-offset was approximately $9 \mu \mathrm{m}$. Figure 3 illustrates the reflection spectrum of the sensing head. It is visible the FBG core reflected mode which is centered in $1552 \mathrm{~nm}$ and the dominant cladding mode which wavelength center is $\sim 1548 \mathrm{~nm}$. This special reflection spectrum results from the cladding-mode-assisted recoupling by the FBG and the core misaligned section.

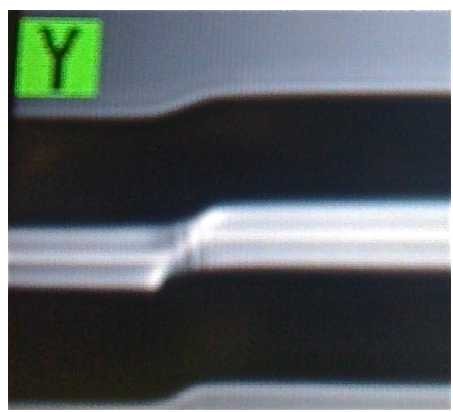

Figure 2 - Splice with a lateral core off-set of $\sim 9 \mu \mathrm{m}$.

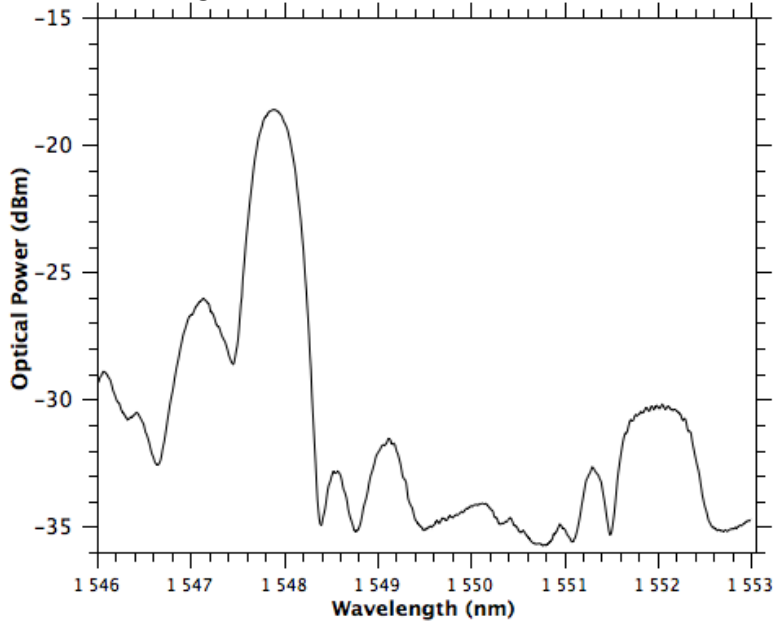

Figure 3 - Reflection spectrum of the sensing head.

For characterization of the sensing head the experimental setup showed in the figure 4 was used. An erbium-doped fiber source illuminated the sensor through an optical circulator which enabled the Fiber Bragg reflected signal to be readout in reflection. Using a Dense Wave-Division-Multiplexer (DWDM) to separate the core reflection from the cladding reflection enabled the optical power to be readout by two photodetectors. The two ends of the fiber containing the sensing probe were fixed on two blocks, being one mounted on a translation stage in order to curve the fiber. The sensing head was placed in the middle of the two blocks, and the resulting curvature $R$ is simply given by the following expression [3], $R=2 h / h^{2}+(d / 2)^{2}$ where $d$ is the distance between the two blocks and $h$ is the bending displacement. The effect of bending on the reflected spectrum of the sensor can be observed on figure 5 .

The measurements were obtained by optical power difference between the cladding and core mode. The curve as function of the curvature radius is showed in figure 6, where a polynomial behavior was observed. Figure 7 illustrates the curve as function of the curvature. The temperature changes did not affect the reflected optical power, which means that through this technique is possible to obtain curvature measurement independent of the temperature [3]. 


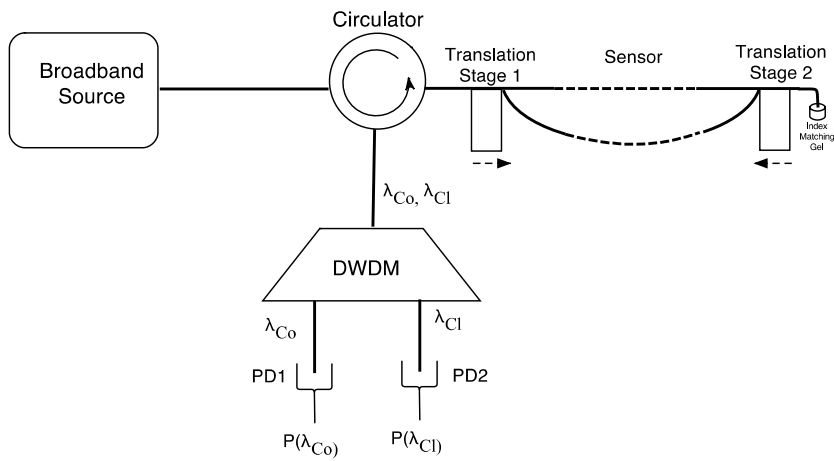

Figure 4 - The experimental setup.

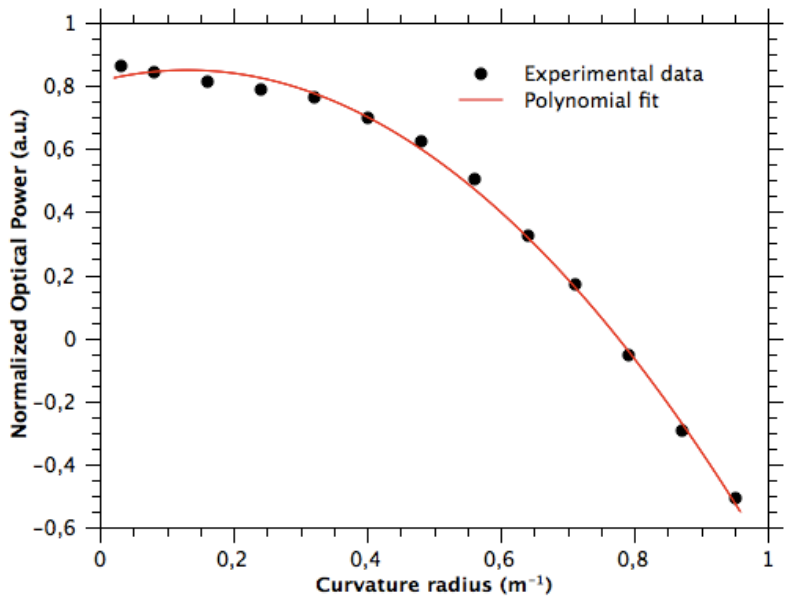

Figure 6 - Normalized optical power as function of the curvature radius.

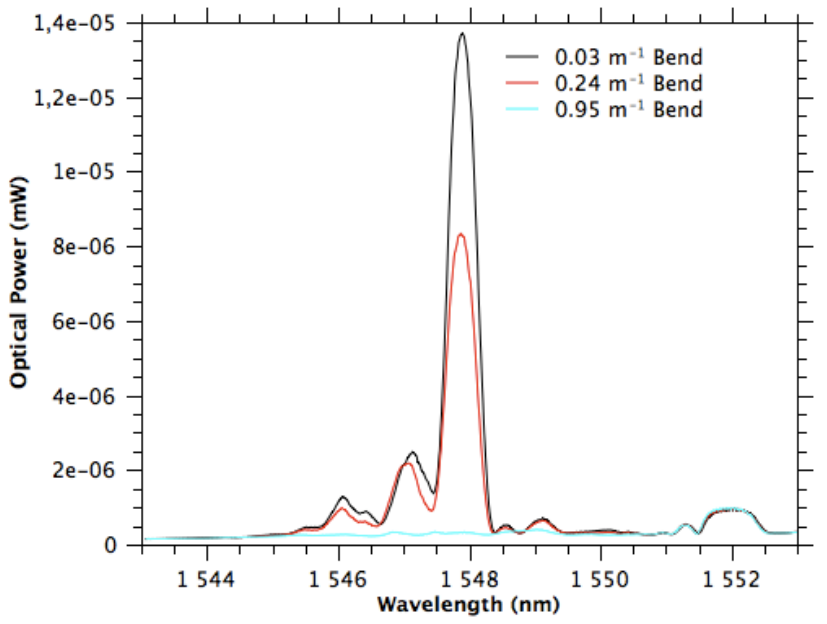

Figure 5 - Spectral behavior of the sensing head for different bending radius.

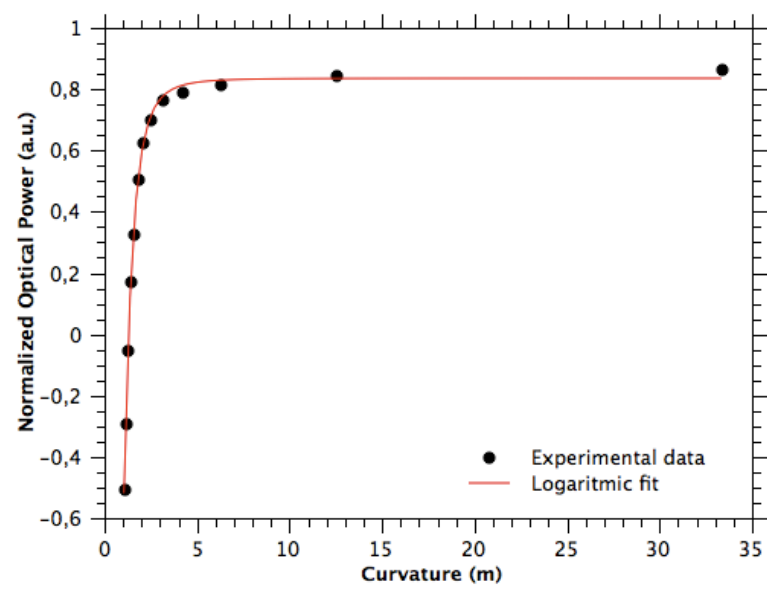

Figure 7 - Normalized optical power as function of the curvature.

\section{CONCLUSION}

In this work a compact and simple curvature sensor system is described. The sensor is based on a simple fiber Bragg grating spliced to a core-offset fiber. This configuration permits to read the cladding mode of the Bragg grating reflected spectrum. The results show that the optical power of the cladding mode is sensitive to fiber curvature, while the core mode is not sensitive. Through the difference of both reflected optical powers, it is possible to obtain an auto-referenced measurement of curvature. This technique is not wavelength dependent avoiding expensive interrogation systems. Moreover, the temperature did not affect the optical power reflected by the sensing head, thus the bending measurement is independent of the temperature.

\section{ACKNOWLEDGEMENTS}

This work was supported by "Fundação para a Ciência e Tecnologia” (PTDC/SAU-BEB/102247/2008, Hybrid). 


\section{REFERENCES}

[1] A. D. Kersey, M. A. Davis, H. J. Patrick, M. LeBlac, K. P. Koo, C. G. Askins, M. A. Putnam, E. J. Friebele, "Fiber Grating Sensors", Journal of Lightwave Technology, Vol. 15, No. 8, 1997.

[2] Yu et al. Bend sensor using an embedded etched fiber Bragg grating. Microw Opt Techn Let (2004) vol. 43 (5) pp. 414-417.

[3] Caucheteur et al. Simultaneous bend and temperature sensor using tilted FBG. 17th International Conference on Optical Fibre Sensors, (2005) vol. 5855 pp. 707.

[4] Jin et al. Temperature-independent bending sensor with tilted fiber Bragg grating interacting with multimode fiber. Opt Communications, (2009) vol. 282 (19) pp. 3905-3907.

[5] Guo et al. Temperature-independent tilted fiber grating vibration sensor based on cladding-core recoupling. Opt Lett, (2008) vol. 33 (9) pp. 1004-1006.

[6] Huang et al. VCSEL-Based Tilted Fiber Grating Vibration Sensing System. IEEE Photonics Technology Letters, IEEE (2010) vol. 22 (16) pp. 1235-1237.

[7] Shao et al. Highly sensitive bend sensor with hybrid long-period and tilted fiber Bragg grating. Optics Communications, (2010) vol. 283 pp. 2690.

[8] Zhang et al. Cladding-mode-assisted recouplings in concatenated long-period and fiber Bragg gratings. Opt Lett, (2002) vol. 27 (14) pp. 1214-1216. 